\title{
Empathy in Social Agents
}

\author{
Ana Paiva \\ INESC-ID and Instituto Superior Técnico, Av. Prof. Cavaco Silva, Porto Salvo, Oeiras, Portugal.
}

\begin{abstract}
Empathy is seen as the capacity to perceive, understand and experience others' emotions. This notion is often considered as one of the major elements in social interactions between humans. As such, when creating social agents, that are believable and able to engage users in social interactions, empathy needs to be addressed. Indeed, for the past few years, many researchers have been looking at this problem, not only in trying to find ways to perceive the user's emotions, but also to adapt to them, and react in an empathic way. This paper provides a small overview of this new challenging area of research, by analyzing empathy in the social relations established between humans and social agents, and providing a concrete model for the creation of empathic social agents.
\end{abstract}

Index Terms-Empathic social agents; emotions in virtual agents.

\section{INTRODUCTION}

Creating social agents that interact with users in natural ways is a new, exciting and challenging field of research. This field addresses several questions involving many different areas of study ranging from the creation of 3D graphics, the generation of intelligent behaviors and the investigation on the social aspects of characters, among others. This is indeed a multidisciplinary endeavor that gathers know-how from researchers in these different areas, working towards the common goal of creating believable social characters. Inspired by Disney [1], the work by Bates [2] in the eighties, established some essential grounds for this field. In particular, it focused the development of these agents not only around their "visual elements" and how realistic characters can be, but also, on their intelligent behaviors. Further, research that deals with the social relations between agents and users need to get inspiration in the social encounters humans establish in their societies. This inspiration led researchers to explore topics such as nor-verbal behaviors, emotions, cultural biases, and other factors. One of these factors is empathy, which is the process associated with the capability of perceiving and reacting to another's emotional state.

This short paper addresses the issue of empathy in social agents, by presenting three cases where empathic agents have been developed and tested.

Manuscript Received on February 2， 2011

E-mail: ana.paiva@inesc-id.pt

\section{EMPATHY IN HUMANS AND IN AGENTS}

According to Hoffman [3], empathy is associated with the psychological processes that make a person have "feelings that are more congruent with another's situation than with his own situation". For example, if one sees another person being robbed in the street, we may feel fear, anger, and distress, although the event has nothing to do with us. In fact, we may approach the person to help or even, try to catch the robber. This type of emotional response is associated with a process known as empathy. According to Hoffman, empathy is the product of natural selection, and is a response aroused by emotional clues coming from another [3], in particular distress cues. On the other hand, F. de Waal [4], a well known primatologist, considers that one can find empathic processes across different species. According to him, empathy can be seen as simple mechanism of synchronization of the bodies: running when the others run, crying when the others cry, and yawning when the others yawn. For example, yawn contagion is associated with the power of synchrony, which is presented not only in us, humans, but also in other animals.

Although many researchers associate empathy with a voluntary process that requires role-taking and higher cognition, according to researchers like de Waal, empathy also embraces non-voluntary processes. As de Waal [4] points out, findings have shown that "we do not decide to be empathic, we just are".

But, when considering the empathic process, there are a set of constructs that need to be considered, namely:

- $\quad$ an observer (or empathizer) who is the person/agent that reacts empathically to the emotions of another one;

- a subject: who is the person/agent that expresses or is associated with an emotional state, perceived by the observer;

- $\quad$ the event or event cue that happens and is witnessed (directly or indirectly) by the observer;

- the emotion: which is felt by the subject and about which the observer empathizes with;

- the situation/context that characterizes the occurrence of an event leading to the emotion. It includes aspects such as the relation between the observer and the subject, the presence or not of other agents, the past situations, etc. 
The situation/context is extremely important as it conditions the whole empathic process and many factors affect it, by intensifying it, modifying or reducing it. We call these factors modulating factors of the empathic process. In fact, it has been observed that even in animals, there is some intensification of empathic experience by the perceived reaction of the others [4]. Further, one of the factors that mediate the empathic process is the identification with the others. Indeed, in order to have empathy we need to identify ourselves with the others in some way. Identification is what makes us adopt the emotions, situation and behavior of the others we are empathizing with. Identification is more strong in moments of high emotion and stronger with people we know well, in particular our close relatives. In fact, we may have a hard time identifying with people whom we see as different or that belong to a different group from ourselves. However, that does not mean that we do not empathize with strangers. In fact, identification can even occur between species. Further, and according to Gaertner and Dovidio [5] the intensity of the observer's empathic arousal is systematically related to the speed of her subsequent helping action.

Given that empathy is such a natural and well studied process found in humans and other species, it should be natural that, as we pursue towards the goal of creating intelligent social agents, we embed such empathic processes in our agents.

But, as pointed out in [6] there are two distinct ways to look at empathy in social agents: one is to consider that the empathizer is the user and the agent is what triggers the empathic process in the user. The second way is to create agents that themselves empathize with other agents, and in particular with the user.

As such, we will consider the following definition of an empathic agent:

\section{Def: "Empathic agents" are agents that respond emotionally} to situations that are more congruent with the user's or another agent's situation, or as agents, that by their design, lead users to emotionally respond to the situation that is more

congruent with the agent's situation than the user's one.

\section{THE CASE OF FEARNOT!}

FearNot! is a learning environment [7] that was developed to help children victims of bullying. The main idea is that a child/user plays the role of a friend of a victim (a synthetic character) and helps the character to deal with the bullying it suffers. This system explores empathy in the autonomous characters as it embeds some constructs that lead the user/child to feel sorry for the victim character and thus, act out in an empathic manner. By exploring the empathy that the user has towards the character, the system aims at helping the children that are victims themselves. Through this safe environment, the child that is victim can test different strategies for acting and coping, and as such learn what strategies to adopt in similar situations.

The system works as follows: a child sees a bullying situation unfolding in a 3D virtual environment, which is populated with other virtual agents. One of them is a victim of bullying and one is a bully. This scenario is generated as a result of the autonomous actions of the autonomous characters that are present in the scene. Once a bullying episode occurs, the child/user is allowed to interact with the victim through a natural language interface, and advise the victim character on what to do next.

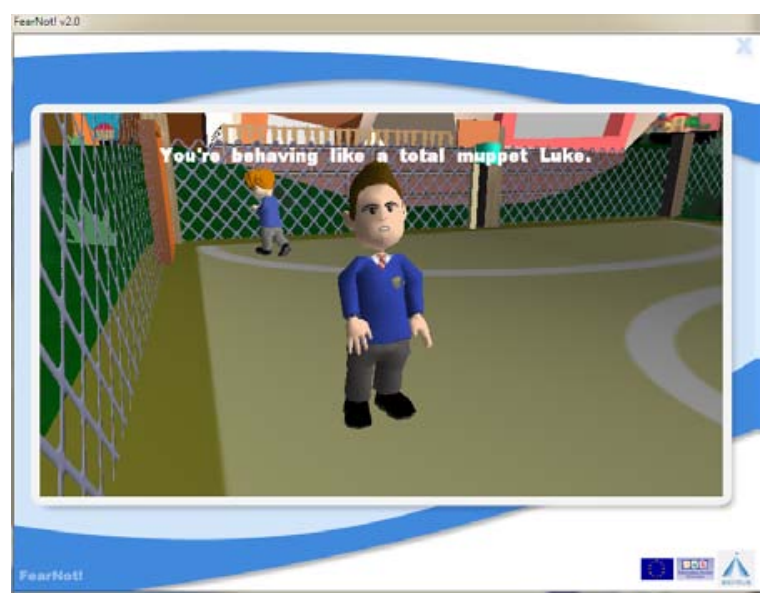

Fig. 1. Screenshot of FearNot! (Color Plate 1)

In FearNot! empathy was explored through the generation of situations with a subject (the victim character), which is lead to exhibit certain emotions (in particular sadness) to situations that arise in the virtual scenario. FearNot!'s agents try to evoke empathic responses from the user, in particular aiming at changing attitudes in children. But to do that, and indeed have an impact on the affective responses of the users, the characters needed to be created and designed in a manner that there was some identification with the characters and with their situations.

In particular we have the following components in the process:

- The observer- in this case is the user (child);

- The subject, which in this case is the virtual character that is victim of bullying episodes;

- The situation- which is generated dynamically in the environment, through the presence of the autonomous characters. Some modulating factors were considered in order to intensify the emotional reaction of the observer/user.

In fact, FearNot! explores deeply these modulating factors, in particular the similarity between the user and the character so that the empathic responses were stronger for the concrete user group we wanted to focus on. Aspects such as the physical appearance and the character's clothes were studied (for example, the characters for the UK schools had uniforms, whereas the characters for the German and Portuguese schools had normal clothes). The gender issue was also considered, and two types of episodes were explored: bullying between females, which involved relational bullying, such as exclusion from parties or games, name calling, etc, and bullying between boys, which is more direct and physical, involving 
punching, hitting etc.

Finally, for the emotions of the subject to be easily recognizable by the users, the facial expressions of the characters were exaggerated and easy to identify (also because the characters were cartoon characters).

A study involving FearNot! was done in the UK and Germany with thousand one hundred twenty-nine children (mean age 8.9) (see [8] for more details of the study). The main goal of the study was to asses if the victims of bullying (which had been identified a-priory) would escape bullying after being exposed to the interaction with FearNot!. In fact, we wanted to explore empathy in a way that would help the child victim to react to the situation using some copping mechanisms. The results obtained after a three-week intervention showed that children that were identified as victims were more likely to stop being victims than the children in a parallel control group's. Although this was a very good result, it was only significant on the first re-assessment, and only for the UK children.

\section{ICAT AS AN EMPATHIC CHESS PLAYER}

The iCat chess player (see [9]) is a scenario built with the iCat robot by Philips, where the robot is able to play chess with users, in particular, children. The iCat can react emotionally to each game situation, like for example, when the child does a very good and unexpected move, it may react by showing some signs of being upset. The affective responses of the iCat are based on its expectations about the next game situation, taking into account what has been played before.

Such initial iCat scenario was extended to a second scenario where the iCat, rather than playing a game, watches two human players doing it, and makes comments to the game being played (see Fig. 2) [10]. For example, if one of the players makes a good move, the iCat may say "That was a good move".

In this scenario, empathy was explored by giving the iCat a different relation to the two different players. In fact, the iCat sees one of the players as his/her companion, and as such, reacts in a very empathic way, providing encouraging comments, and being enthusiastic towards his/her moves. On the other hand, to the other player, the iCat's relation is mostly neutral, and as such, the comments it does are mainly factual and less emotive.

For example, consider the situation where the iCat's companion unexpectedly loses one important piece in the game and his/her situation is now much worse than before. In this case the iCat puts itself in the position of his companion and appraises the situation as if it would have happened to himself (an empathic appraisal process). Yet, towards the other player, the iCat reacts in a neutral way, simply acknowledging his/her good move. Differently, if the appraised situation has a positive valence for his companion, the iCat reacts with a positive feedback (as it would feel itself), becoming happy and thus praising his companion.

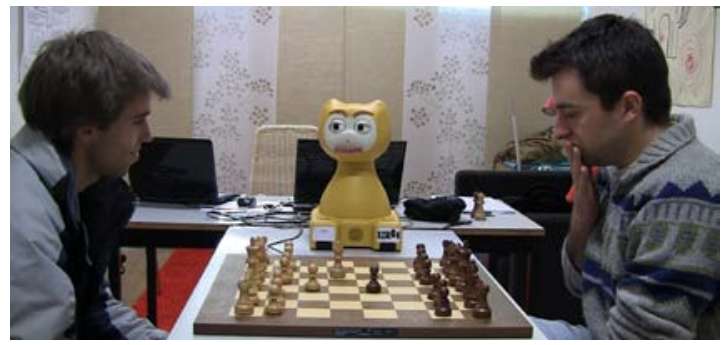

Fig. 2. The iCat as an empathic companion

In terms of emotional responses, the iCat uses not only verbal responses but also emotional facial expressions to accompany the feedback.

In this concrete case studied, the observer/empathizer is the iCat and the subject is the iCat's companion. However, the emotions of the subject are not captured directly by the iCat but are predicted as the possible emotions through the affective modeling of the user, using a mechanism of perspective taking.

This scenario, although simple, was evaluated through an experiment conducted with 40 subjects with ages ranging from 18 to 28 years old. Each participant played a chess game against another participant. No-one knew the final goal of the experiment nor that the iCat was responding differently to the two players. At the end of the game, the participants filled a questionnaire assessing their degree of "friendship" to the iCat, using the McGill Friendship questionnaire [11]. The results showed that users to whom the iCat was empathic, preferred significantly to interact with it, as they found it more enjoyable, and more importantly they would consider to spend more time interacting with it than the others to whom the iCat was not empathic.

\section{A PROCESS MODEL OF EMPATHY}

Although most of the work done of empathic for social agents has involved the user as the empathizer (as in the case of FearNot!) or as an observer (as in the case of the iCat), we can however create scenarios of empathy to simulate the dynamics that emerge in the social interactions between artificial agents in synthetic societies, thus not involving the user directly. But in order to do so, a concrete model for an empathic agent must be built.

In [12] a generic process model of empathy was proposed and implemented in a computational framework. Such model considers that empathy is a process that involves two stages: the empathic appraisal and the empathic response. The first one considers the appraising of events where the subject has a concrete emotional state, as if the events happened to observer/empathizer. The second phase in the process consists in the generation of behavioral reactions to the appraised situation.

The model considers also that there are a few modulating factors that condition the empathic emotion. These modulating factors are: the affective link between the subject and the empathizer; the similarity between them two and the mood and personality of the empathizer. Fig. 3 shows the 
model proposed and implemented through an agent architecture.

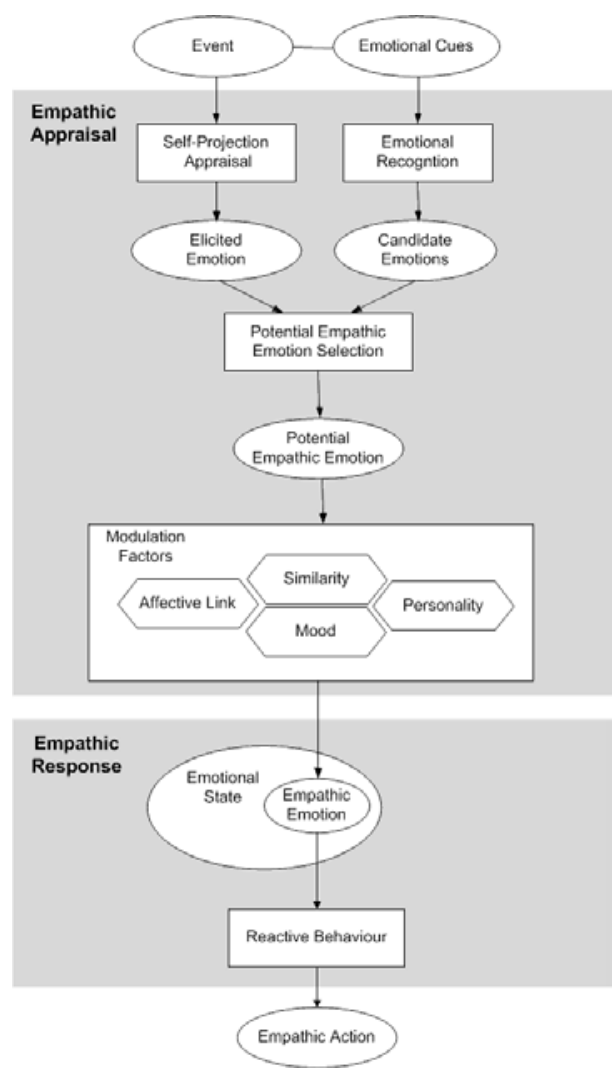

Fig. 3. The model implemented through an agent architecture.

This model was used in a small scenario with 4 agents and was subsequently used for an evaluation to test the impact that the model had on the behaviour of the agents. To evaluate the model two conditions were developed: one with the empathy model and one without it. In both cases the agents had the same goals, personality and roles in the scenario. The results of the evaluation showed that the behavior of the agents was well perceived by the viewers of the simulation and that the agents with empathy were perceived as more caring and likeable.

\section{CONCLUDING REMARKS}

This paper provides an overview of a set of empathic agents, analyzes under the light of a framework that considers empathy as a dynamic process involving several constructs.

This area has had a large advance in the past few years, but there is still a lot of work to be done, not only in trying to achieve social agents that are able to better understand the user's emotional states and respond emotionally to them, but also, in creating models and simulations that show the emergence of some social emergent phenomena in human societies as a result of better empathy models.

\section{ACKNOWLEDGEMENTS}

The research leading o these results has received funding from the European Community's Seventh Framework Program (FP7/2007-2013), under the grants agreement for the LIREC and the Ecute projects, plus from the FCT - Portuguese Science Foundation (INESC-ID multiannual funding) through the PIDDAC Program funds.

\section{REFERENCES}

[1] O. Johnston and F. Thomas, "The Illusion of Life: Disney animation”, Disney Editions, 1995.

[2] J. Bates, "The Role of Emotion in Believable Agents", in Communications of ACM., vol. 37(7), ACM Press, 1994.

[3] M. Hoffman, Empathy and Moral Development, Cambridge University Press, 2000

[4] F. de Waal, “The age of Empathy”, Harmony Books, 2009.

[5] Gaertner, Samuel L.; Dovidio, John F., The subtlety of White racism, arousal, and helping behavior, in Journal of Personality and Social Psychology, 35(10), 1977, 691-707.

[6] A. Paiva, et. al. "Caring for Agents and Agents that Care: Building empathic relations with synthetic agents”, AAMAS 2004, ACM Press, 2004.

[7] A. Paiva et. al. Learning by feeling: Evoking empathy with synthetic characters, Applied Artificial Intelligence, vol. 16, n. 3-4. 2005.

[8] M. Sapouna et. al. "Virtual learning intervention to reduce bullying victimization in primary school: a controlled trial”, Journal of Child Psychology and Psychiatry 51:1 (2010),

[9] I. Leite, C. Martinho, A. Pereira and A. Paiva, “As Time goes by: Long-term evaluation of social presence in robotic companions” in Proceedings of the 18th IEEE International Symposium on Robotics RO-MAN 2009, IEEE Computer Society, 2009.

[10] I. Leite et. al. "Why Can't We Be Friends?" An Empathic Game Companion for Long-Term Interaction, in Proceedings of the 10th International Conference on Intelligent Virtual Agents, IVA'2010, Springer Berlin / Heidelberg, 2010.

[11] L. K. Souza “Amizade em adultos: adaptação e validação dos questionários McGill e um estudo de diferenças de género (in Portuguese)”, PhD thesis, Universidade Federal do Rio Grande do Sul, 2006.

[12] S. Rodrigues, S. Mascarenhas, J. Dias and A. Paiva, "I can feel it too! Emergent empathic reactions between synthetic characters" in Affective Computing and Intelligent Interaction Conference, ACII, IEEE Press, 2009.

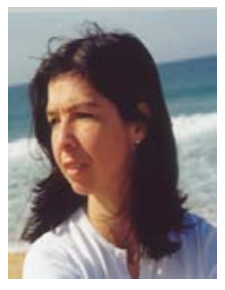

Ana Paiva is an Associate Professor at Instituto Superior Técnico, Technical University of Lisbon and the GAIPS research group leader at INESC-ID. She is well known in the area of Intelligent Agents, Virtual Agents, Affective Computing and Artificial Intelligence Applied to Education.

Her research is focused on the affective elements in the interactions between users and computers and in particular in the creation of affective behaviour in

synthetic virtual agents.

Prof. Ana Paiva served as a member of numerous international conferences and workshops. She has (co)authored over 120 publications in refereed journals, conferences and books and coordinated the participation of INESC-ID in many international and national research projects. Prof. Ana Paiva is member of the IEEE, ACM, and AAAI. 